\title{
Evaluation of the Role of Hyaluronic Acid as A potential Biomarker for Diagnosis of Non-Alcoholic Fatty Liver Disease
}

Ahmed Mohamed ElGhandour*, Emad Ahmad Awad, Riham Hamed Ahmed Abdel-Hamid, Ahmed M. F. Mansour

Gastroenterology, Hepatology and Internal Medicine Department, Ain Shams University, Egypt *Correspondence author: Ahmed Mohamed ElGhandour, Mobile: 002 01001973493, E-mail: ghandour80@ hotmail.com

\begin{abstract}
:
Background: Non-alcoholic fatty liver disease (NAFLD) is the most common chronic liver disease worldwide, ranging from simple steatosis and non-alcoholic steatohepatitis (NASH) to cirrhosis, with its complications including hepatocellular carcinoma (HCC). The non-invasive diagnostic markers of NAFLD are gaining interest due to the invasive nature and potential side effects of liver biopsy, and are the current gold standard of diagnosis. Objective: This study aimed to evaluate hyaluronic acid (HA) as a potential non-invasive biomarker for diagnosis and prognosis of NAFLD and to compare it with the traditional non-invasive techniques.

Patients and methods: This study included 63 subjects divided into 3 groups; including 21 patients with fatty liver, 21 patients with NASH, in addition to 21 healthy controls. Non-invasive assessment of liver fibrosis was done to all study subjects using aspartate aminotransferase to platelet ratio index (APRI) and fibrosis-4 score (FIB4), in addition to measurement of HA serum levels.

Results: As regard assessment of liver fibrosis, there was a highly significant statistical difference between groups as regard APRI and Fib4 scores. As for HA there was a highly significant statistical difference between study groups. HA receiver operating characteristics curves (ROC) achieved excellent diagnostic performance for fatty liver with $100 \%$ sensitivity and specificity at cutoff of $25 \mathrm{ng} / \mathrm{ml}$ for fatty liver, versus a sensitivity of $95.2 \%$ and specificity of $85.7 \%$ for NASH at cutoff of $110 \mathrm{ng} / \mathrm{ml}$.
\end{abstract}

Conclusion: HA can be used as an accurate and specific non-invasive biomarker for the diagnosis of NAFLD and staging of the severity of disease compared with the traditional known non-invasive scores.

Keywords: Biomarker, Hyaluronic Acid, Non-Alcoholic Fatty Liver Disease.

\section{INTRODUCTION}

NAFLD is the most prevalent chronic liver disease worldwide, and it occurs in every age group, but particularly during the fourth and fifth decades of life, especially in patients with risk factors such as hyperglycemia and hyperlipidemia in the presence of a high body mass index (BMI). NAFLD is a multisystem disease that affects not only the liver, but also organs outside the liver. It. contributes to an increased risk of cardiovascular disease, chronic kidney disease, and type 2 diabetes mellitus (DM). For this reason, the mortality rate increases by $57 \%{ }^{(1)}$.

NAFLD refers to the full range of fatty liver diseases from simple steatosis to NASH, advanced fibrosis and cirrhosis ${ }^{(2)}$. The common pathway leading to liver fibrosis and cirrhosis is growing deposition of extracellular matrix. The main structural role in the formation of the extracellular matrix is played by HA, which has been found to be associated with the histological stages of liver disease ${ }^{(3)}$.

Staging and diagnosing the severity of liver disease is done by either an invasive or a non-invasive approach. The invasive method is through taking a liver biopsy, which is so far the gold standard for diagnosis of NAFLD. However, liver biopsy as an invasive technique carries risks of many complications e.g. bleeding and infection, besides being expensive and not suitable for screening all
NAFLD patients. Subsequently, several non-invasive markers have been evaluated for the diagnosis of NAFLD including both serological indices and imaging methods. A large number of algorithms have been developed for differentiating between simple steatosis and NASH including: APRI ${ }^{(4)}$, FIB-4 score, which are so far the most prominent scoring systems for distinguishing NASH from simple steatosis. Both of these algorithms depend on Age, aspartate aminotransferase (AST), alanine aminotransferase (ALT), and platelet count. Other scoring systems include: Hepatic Steatosis Index (gender, DM, body mass index "BMI", ALT, AST), Bard score (BMI, AST/ALT ratio, DM), NAFLD Fibrosis Score (Age, BMI, DM, platelet count, albumin, AST/ALT ratio), Nippon Score (gender, age, DM, hypertension) ${ }^{(5)}$ and Fatty Liver Index (an algorithm based on BMI, waist circumference, triglycerides and gamma-glutamyl transferase "GGT") (6). Though, these scoring systems have some limitations. They have a limited ability to detect the early stages of liver disease, and they have a high false positive rate in advanced fibrosis ${ }^{(7)}$.

According to the previous limitations, there is a growing need for evaluation of a new non-invasive dependable biomarker for diagnosis of NAFLD and assessment of its severity. HA is a high molecular weight polysaccharide that is practically found in every tissue in the body and is synthesized in synovial 
lining cells and hepatic stellate cells by an enzyme called HA synthase. ${ }^{(8)}$. Production of HA initially increases when collagen synthesis is accelerated under various inflammatory conditions and liver cell injury, resulting in raised serum levels of HA. Consequently, serum HA concentration reflects the severity of liver damage, which makes it a valuable non-expensive, simple and quick biomarker for staging of NAFLD ${ }^{(9)}$.

The aim of this study was to evaluate HA as a potential non-invasive biomarker for diagnosis and prognosis of NAFLD, and to compare it with the traditional non-invasive techniques for assessment of liver fibrosis.

\section{PATIENTS AND METHODS}

This study was conducted on sixty-three individuals through the period from March to September 2020. They were recruited from the Gastroenterology and Hepatology Department of Ain Shams University Hospital and divided into three different groups as following: 21 patients with fatty liver, 21 patients with NASH, in addition to 21 healthy controls.

The study included patients with a high BMI (>25 $\mathrm{kg} / \mathrm{m}^{2}$ ), bright/fatty liver on abdominal ultrasonography, presence or absence of abnormalities in liver enzymes, and after exclusion of other causes of liver disease.

All individuals were subjected to thorough history taking, clinical examination, abdominal ultrasonography and routine laboratory investigations to assess liver functions and exclude other causes of liver disease. Serum HA level was assessed using enzyme-linked immunosorbent assay (ELISA) technique.

\section{Ethical approval}

An approval of the study was obtained from Ain Shams University academic and ethical committee. Every patient signed an informed written consent for acceptance of the operation.

\section{Statistical Analysis}

Statistical presentation and analysis of the results from the present study was conducted using SPSS version 23 computer software. The data were described using frequency, mean, standard deviation, and range and expressed in tables and figures. Data were compared using one-way analysis of variance (ANOVA) test, and chi-square. The cutoff level of hyaluronic acid with the highest sensitivity and specificity rates was chosen using the receiver operating characteristic (ROC) curve. A p-value less than 0.05 was considered statistically significant.

\section{RESULTS}

There was no statistically significant difference between the three groups as regard sex. As regard age there was statistically significant difference between the three groups. Our results showed that there was statistically significant difference between the study groups as regard BMI (Table 1).

Table (1): Demographic data and comparison between the study groups according to BMI.

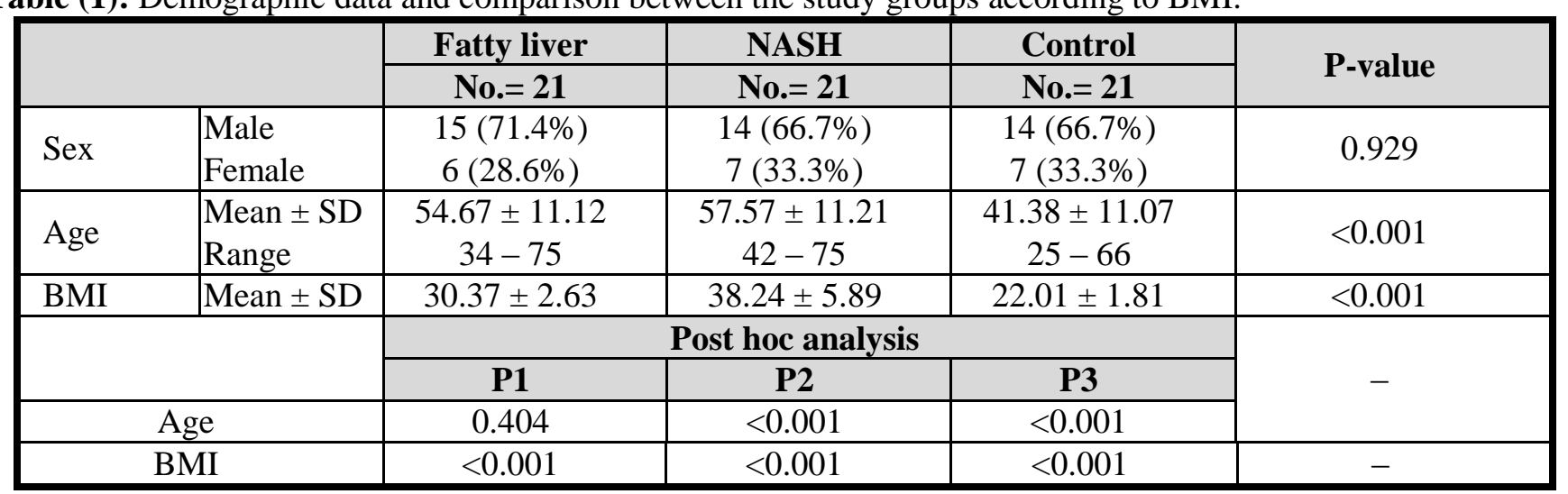

P1: Fatty liver VS NASH P2: Fatty liver VS Control

P3: NASH VS Control

As regard liver functions, there was high statistically significant difference between the three groups as regard platelets count as the mean count was significantly lower in NASH group than other two groups. As regard bilirubin there was statistically significant difference fatty liver and NASH group and between NASH and control group with higher mean bilirubin in NASH group and nearly high normal in fatty liver group and normal in control group. Also there was statistically significant difference between NASH and fatty liver group with the mean level lower in NASH group as regard albumin. ALT and AST were higher in NASH group with statistically significant difference, but there was no statistically significant difference between the groups as regard INR (Table 2).

Table (2): Comparison between the three study groups according to liver function tests

\begin{tabular}{|l|c|c|c|c|l|}
\hline \multicolumn{2}{|c|}{} & Fatty liver & NASH & Control & \multirow{2}{*}{ P-value } \\
\cline { 3 - 6 } \multicolumn{2}{|c|}{} & No.= 21 & No.= 21 & No.= 21 & \\
\hline PLT & Mean \pm SD & $232.24 \pm 7.41$ & $172.38 \pm 17.86$ & $301.62 \pm 41.67$ & $<0.001$ \\
\hline
\end{tabular}




\begin{tabular}{|c|c|c|c|c|c|}
\hline \multicolumn{6}{|l|}{ x $10^{9} / \mathrm{L}$} \\
\hline INR & Mean \pm SD & $0.95 \pm 0.12$ & $0.96 \pm 0.16$ & $0.93 \pm 0.11$ & 0.842 \\
\hline $\begin{array}{l}\text { Albumin } \\
\mathrm{g} / \mathrm{dl}\end{array}$ & Mean \pm SD & $4.25 \pm 0.5$ & $3.78 \pm 0.41$ & $4.06 \pm 0.48$ & 0.007 \\
\hline $\begin{array}{l}\text { Bilirubin } \\
\mathrm{mg} / \mathrm{dl}\end{array}$ & Mean \pm SD & $0.71 \pm 0.1$ & $1.13 \pm 0.07$ & $0.64 \pm 0.3$ & $<0.001$ \\
\hline $\begin{array}{l}\mathrm{AST} \\
\mathrm{IU} / \mathrm{L}\end{array}$ & Mean \pm SD & $25.9 \pm 2.56$ & $219.05 \pm 6.9$ & $25.43 \pm 13.35$ & $<0.001$ \\
\hline $\begin{array}{l}\text { ALT } \\
\text { IU/L }\end{array}$ & Mean \pm SD & $35.76 \pm 6.03$ & $290.95 \pm 36.18$ & $23.71 \pm 10.49$ & $<0.001$ \\
\hline \multicolumn{5}{|c|}{ Post hoc analysis } & \multirow{8}{*}{-} \\
\hline & & P1 & P2 & P3 & \\
\hline \multicolumn{2}{|c|}{ PLT } & $<0.001$ & $<0.001$ & $<0.001$ & \\
\hline \multicolumn{2}{|c|}{ INR } & 0.829 & 0.692 & 0.578 & \\
\hline \multicolumn{2}{|c|}{ Albumin } & 0.002 & 0.228 & 0.048 & \\
\hline \multicolumn{2}{|c|}{ Bilirubin } & 0.002 & 0.429 & $<0.001$ & \\
\hline \multicolumn{2}{|c|}{ AST } & $<0.001$ & 0.906 & $<0.001$ & \\
\hline \multicolumn{2}{|c|}{ ALT } & $<0.001$ & 0.006 & $<0.001$ & \\
\hline
\end{tabular}

INR: International Normalization Ratio

P1: Fatty liver VS NASH P2: Fatty liver VS Control

P3: NASH VS Control

Moreover, this study showed that there was a highly significant statistical difference between the study groups as regard cholesterol, LDL, HDL and triglycerides (Table 3).

Table (3): Comparison between the three study groups as regard lipid profile

\begin{tabular}{|c|c|c|c|c|c|}
\hline & \multirow{2}{*}{$\begin{array}{c}\text { Fatty liver } \\
\text { No.= 21 } \\
\end{array}$} & \multirow{2}{*}{$\begin{array}{c}\text { NASH } \\
\text { No. }=21 \\
\end{array}$} & \multirow{2}{*}{$\begin{array}{l}\text { Control } \\
\text { No. }=21 \\
\end{array}$} & \multirow{2}{*}{ P-value } \\
\hline & & & & & \\
\hline $\begin{array}{l}\text { Cholesterol } \\
\mathrm{mg} / \mathrm{dl}\end{array}$ & Mean \pm SD & $198.81 \pm 37.48$ & $263.57 \pm 51.07$ & $115.95 \pm 24.17$ & $<0.001$ \\
\hline $\begin{array}{l}\mathrm{LDL} \\
\mathrm{mg} / \mathrm{dl}\end{array}$ & Mean \pm SD & $146.10 \pm 11.39$ & $192.38 \pm 13.93$ & $81.43 \pm 11.53$ & $<0.001$ \\
\hline $\begin{array}{l}\mathrm{HDL} \\
\mathrm{mg} / \mathrm{dl}\end{array}$ & Mean \pm SD & $48.10 \pm 8.21$ & $30.29 \pm 6.72$ & $68.76 \pm 8.57$ & $<0.001$ \\
\hline $\begin{array}{l}\text { Triglycerides } \\
\mathrm{mg} / \mathrm{dl}\end{array}$ & Mean \pm SD & $196.67 \pm 21.76$ & $362.86 \pm 9.01$ & $108.67 \pm 19.21$ & $<0.001$ \\
\hline & & & Post hoc analys & & \\
\hline & & P1 & P2 & P3 & \\
\hline Chole & & $<0.001$ & $<0.001$ & $<0.001$ & \\
\hline $\mathrm{LD}$ & & $<0.001$ & $<0.001$ & $<0.001$ & - \\
\hline $\mathrm{HL}$ & & $<0.001$ & $<0.001$ & $<0.001$ & \\
\hline Triglyc & des & $<0.001$ & $<0.001$ & $<0.001$ & \\
\hline
\end{tabular}

LDL: Low-Density Lipoproteins

P1: Fatty liver VS NASH P2: Fatty liver VS Control P3: NASH VS Control

Regarding RBS, there was highly statistically significant difference between the groups (Table 4).

Table (4): Comparison between the three studied groups as regard to random blood sugar (RBS)

\begin{tabular}{|l|c|c|c|c|}
\multirow{2}{*}{ RBS } & Fatty liver & NASH & Control & \multirow{2}{*}{ P-value } \\
\cline { 2 - 4 } & No. $=21$ & No. $=21$ & No.=21 & \\
\hline
\end{tabular}




\begin{tabular}{|l|c|c|c|c|}
\hline \multirow{2}{*}{ Mean \pm SD } & $194.29 \pm 5.92$ & $265 \pm 7.11$ & $100 \pm 7.18$ & $<0.001$ \\
\hline \multirow{3}{*}{} & \multicolumn{3}{|c|}{ Post hoc analysis } & \multirow{2}{*}{-} \\
\cline { 2 - 4 } & P1 & P2 & P3 & $<0.001$ \\
\cline { 2 - 5 } & 0.001 & $<0.001$ & \\
\hline
\end{tabular}

P1: Fatty liver VS NASH P2: Fatty liver VS Control

P3: NASH VS Control

As regard assessment of liver fibrosis, our study showed that there was a highly statistically significant difference between the study groups as regard APRI and Fib4 (Table 5).

Table (5): Comparison between the three study groups as regard to APRI and FIB4

\begin{tabular}{|c|c|c|c|c|c|}
\hline & & Fatty liver & NASH & Control & \multirow{2}{*}{ P-value } \\
\hline & & No. $=21$ & No. $=21$ & No. $=21$ & \\
\hline APRI & Mean \pm SD & $0.29 \pm 0.05$ & $3.21 \pm 0.3$ & $0.21 \pm 0.01$ & $<0.001$ \\
\hline \multirow[t]{3}{*}{ Fib4 } & Mean \pm SD & $1.04 \pm 0.05$ & $4.43 \pm 0.76$ & $0.77 \pm 0.02$ & $<0.001$ \\
\hline & & \multicolumn{3}{|c|}{ Post hoc analysis } & \multirow{4}{*}{-} \\
\hline & & P1 & P2 & P3 & \\
\hline \multicolumn{2}{|c|}{ APRI } & $<0.001$ & 0.063 & $<0.001$ & \\
\hline \multicolumn{2}{|c|}{ Fib4 } & $<0.001$ & 0.044 & $<0.001$ & \\
\hline
\end{tabular}

P1: Fatty liver VS NASH P2: Fatty liver VS Control

P3: NASH VS Control

Additionally, as regard HA, our study showed a highly significant statistical difference between the study groups (Table 6).

Table (6): Comparison between the study groups as regard HA levels

\begin{tabular}{|c|c|c|c|c|}
\hline & Fatty liver & NASH & Control & \multirow{2}{*}{ P-value } \\
\hline & No. $=21$ & No. $=21$ & No. $=21$ & \\
\hline \multirow[t]{4}{*}{$\begin{array}{l}\text { H.A. } \mathrm{ng} / \mathrm{ml} \\
\text { Mean } \pm \text { SD }\end{array}$} & $82.14 \pm 8.4$ & $302.86 \pm 7.04$ & $16.9 \pm 4.7$ & $<0.001$ \\
\hline & \multicolumn{3}{|c|}{ Post hoc analysis } & \multirow{3}{*}{-} \\
\hline & P1 & P2 & P3 & \\
\hline & $<0.001$ & $<0.001$ & $<0.001$ & \\
\hline
\end{tabular}

P1: Fatty liver VS NASH P2: Fatty liver VS Control

P3: NASH VS Control

Evaluation of diagnostic performance of HA using ROC curves showed 100\% sensitivity and specificity for HA for diagnosis of fatty liver at cutoff value $>25 \mathrm{ng} / \mathrm{ml}$. On the other hand, HA showed a sensitivity of $95.2 \%$ and a specificity of $85.7 \%$ for diagnosis of NASH at a cutoff value $>110 \mathrm{ng} / \mathrm{ml}$ (Table 7 and figures 1 and 2).

Table (7): Statistical analysis of HA levels between fatty liver and NASH patients for ROC curve

\begin{tabular}{|c|c|c|c|c|c|c|}
\hline Disease & Cut of point & AUC & Sensitivity & Specificity & +PV & -PV \\
\hline Fatty Liver & $>25 \mathrm{ng} / \mathrm{ml}$ & 100.0 & 100.0 & 100.0 & 100.0 & 100.0 \\
\hline NASH & $>110 \mathrm{ng} / \mathrm{ml}$ & 0.971 & 95.2 & 85.7 & 87 & 49.7 \\
\hline
\end{tabular}

PV: Predictive value 


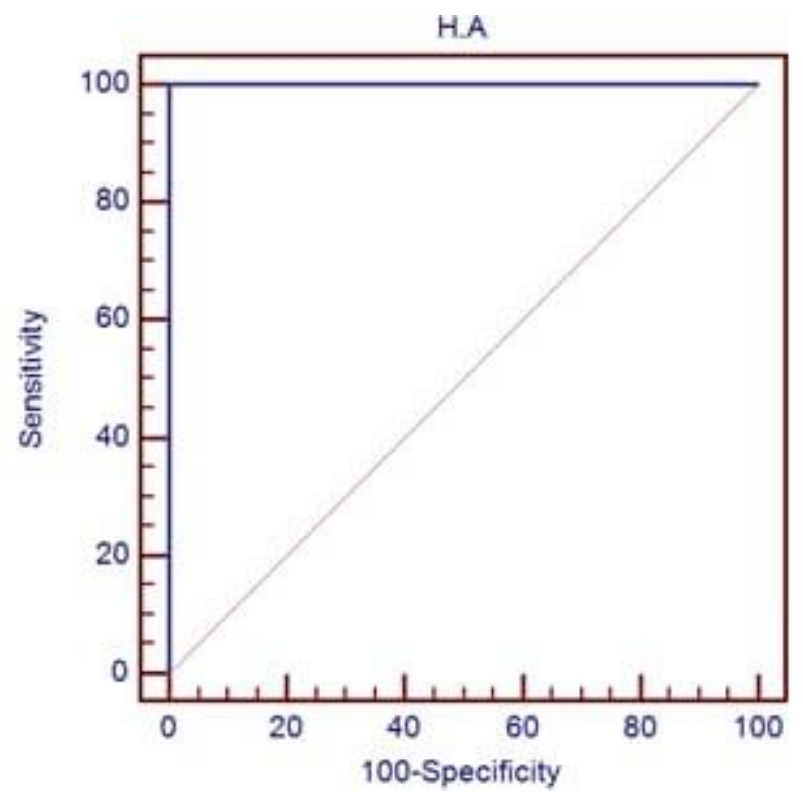

Figure (1): ROC curve (Receiver operator characteristic curve); Accuracy (area under ROC curve) showed 100\% sensitivity and specificity for HA for diagnosis of fatty liver

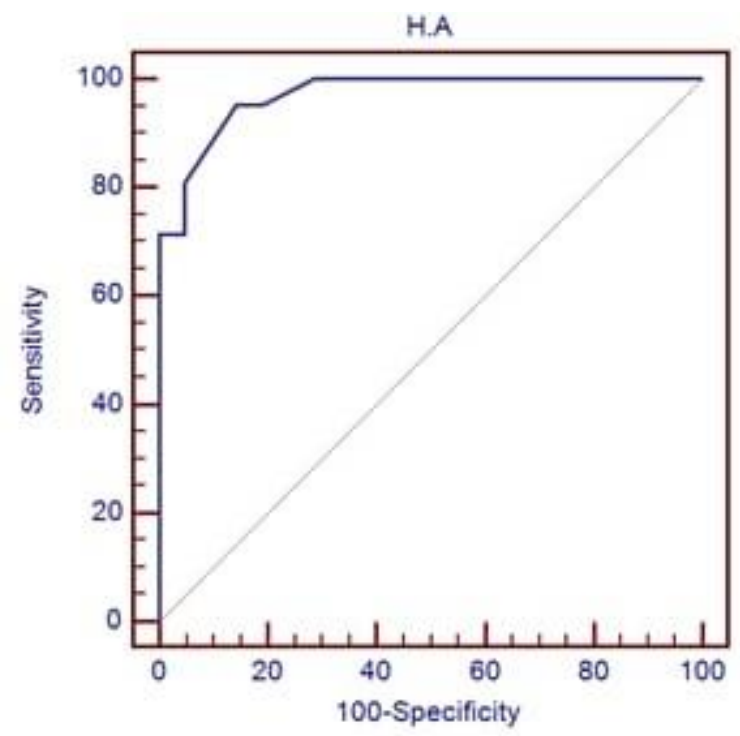

Figure (2): ROC curve (Receiver operator characteristic curve); Accuracy (area under ROC curve) showed $95.2 \%$ sensitivity and specificity of $85.7 \%$ for HA for diagnosis of NASH

\section{DISCUSSION}

NAFLD is one of the most common chronic liver diseases in many developed countries. It is also a serious health problem worldwide. This disease includes a wide range of liver disorders from simple fat accumulation in hepatocytes (simple steatosis) to hepatitis and fibrosis (steatohepatitis) and finally cirrhosis of the liver and even liver cancer. The prevalence of this disease has increased dramatically in past years, and various studies have reported a doubling of its prevalence within 10 years in several countries ${ }^{(\mathbf{1 0})}$.

One of the promising non-invasive biomarkers that have been found to diagnose grade NAFLD is serum HA level. The serum HA level has been shown to be significantly higher in patients with steatohepatitis, compared to healthy controls, and it is also higher in cirrhotic patients than in NASH patients (3). Hence, it is tested as a simple non-invasive biomarker of the degree of fibrosis and in prediction of level of cirrhosis in NAFLD. The accuracy of HA in diagnosing the degree of NAFLD is discussed in this study.

In the current study, we found that there was a highly significant statistical difference between groups as regard ALT, AST, and bilirubin. This agrees with the results of Martin-Rodriguez et al. ${ }^{(11)}$ who correlated the relation between ALT and degree of NAFLD. The results of his study confirmed that serum ALT and AST values were positively correlated with liver fat content and enabled the detection of most of the participants with NAFLD. Liver fat content was highly significantly and 
positively correlated with serum ALT $(r=0.58, p<0.001)$, serum AST $(r=0.32, \mathrm{P}<0.01)^{(11)}$.

Conversely, the results of the current study disagreed with the study of Khosravi et al. ${ }^{(12)}$ who found that ALT levels have little contribution to predict severity of NAFLD. In his study, ALT activity was classified into "normal" and "elevated" according to the reference laboratory cut-off value of $35 \mathrm{U} / \mathrm{L}$. His findings demonstrated that, by considering the current cut-off value of $35 \mathrm{U} / \mathrm{L}$, only 22 patients with NAFLD had normal ALT $(<35 \mathrm{U} / \mathrm{L})$, while the remaining 125 patients had elevated ALT ( $>35 \mathrm{U} / \mathrm{L})$. In spite of that, according to the ROC curve of his study, ALT had a low specificity and sensitivity in predicting NAFLD. In his study, even 30\% of those patients who had elevated ALT, had normal values on the repeated testing. In addition to that, occurrence of significant fibrosis in NASH might be seen in patients with NAFLD who had a normal ALT value. According to him, a retrospective study of 51 patients with normal ALT levels showed bridging fibrosis in 12 and cirrhosis was found in six patients.

All these results, according to his study, underline the little contribution of ALT as an independent factor for detecting the severity of NAFLD. Therefore, using ALT levels as an indicator of severity might result in false reassurance of patients and physicians ${ }^{(\mathbf{1 2})}$. In our opinion, a possible explanation for his study's findings is that those were inaccurate reports due to the implementation of unsuitable ALT cut-off values leading to inaccurate categorization of patients into fatty liver or NASH groups.

We also found in our study that there was a highly significant statistical difference between study groups as regard random blood sugar (RBS) $(\mathrm{p}<0.001)$. This result agrees with the study of Zubair et al. ${ }^{(13)}$ who reported that diabetic patients are at strong risk for both NAFLD and NASH and there is a significant association of fatty liver with DM. Another study explained that the close association between NAFLD and type 2 DM lies in that they have a common pathogenesis, which is insulin resistance. NAFLD can reduce the inhibitory effect of insulin on endogenous glucose production, and reduce the body's insulin sensitivity, making it difficult to control blood glucose in patients with type $2 \mathrm{DM}^{(\mathbf{1 4})}$.

Additionally, we found that that there was a highly significant statistical difference between groups as regard LDL. This agreed with the study of Mahaling et al. (15), who observed that increasing grades of NAFLD were significantly associated with increasing levels of LDL ( $p$-value <0.001).

Continuing the results of our study, there was a highly statistically significant difference between study groups as regard APRI and Fib4. This result agreed with the study of Dyson et al. ${ }^{(16)}$. According to their study, the FIB4 score appears to be one of the most useful non-invasive tests for diagnosing advanced fibrosis in NAFLD. For stage 3-4 fibrosis, a score $<1.3$ has a $90 \%$ negative predictive value (NPV) and a score $>2.67$ has an $80 \%$ positive predictive value (PPV), with $72 \%$ of patients scoring below 1.3 or above 2.67 .

Other studies have confirmed that the FIB4 score is slightly better than other non-invasive tests in diagnosing advanced fibrosis in NAFLD. One of these studies is that of Yousaf $\boldsymbol{e t}$ al. ${ }^{(17)}$ who also suggested that the FIB4 score can provide a definitive diagnosis of NASH with $67 \%$ sensitivity and $73 \%$ specificity. The same study also concluded that FIB4 is also well equipped to identify NASH with mild fibrosis out of a NAFLD study population (57\% sensitivity, and $75 \%$ specificity). A recent study determined the cutoff value of FIB4 score for evaluation of advanced fibrosis. A cutoff value of $<1.45$ excludes advanced fibrosis and has $74 \%$ sensitivity, $71 \%$ specificity, $22 \%$ PPV, $73 \%$ NPV giving an area under the ROC curve (AUROC) of 0.87. Similarly, a higher cutoff value $>3.25$ predicts advanced fibrosis and has $26 \%$ sensitivity, $98 \%$ specificity, $75 \%$ PPV, $85 \%$ NPV giving an AUROC of 0.88 .

Kupčová et al. ${ }^{(18)}$ also showed that FIB4 is the most promising diagnostic panel for distinguishing NASH from steatosis. The greatest AUROC value for the FIB4 index is 0.96 and specificity of $98 \%$ was found to be superior to APRI scoring system with AUROC 0.75 with specificity of $86 \%$ for differentiating between advanced and mild fibrosis in 576 Japanese biopsy-proven NAFLD patients. Using the FIB4 index, $58 \%$ of liver biopsies could be avoided $^{(17)}$.

However, not all studies support this opinion; Zhang et al. ${ }^{(14)}$ had a different point of view. According to their study of 634 patients, the FIB4 algorithm exhibited low specificity in individuals who were older than 65 years. Conclusively, he is suggesting that age might affect the accuracy of this diagnosis and hence it is not an accurate marker.

Besides, there was a highly significant statistical difference between study groups as regard HA level. This agrees with the study of Hadizadeh $\boldsymbol{e t}$ al. ${ }^{(10)}$, who found that with the increase of fibrosis by one degree, there is an accompanied increase of serum level of HA by $40 \%$. Sensitivity, specificity and PPV of this marker were calculated as 100\%, 89\% and $77 \%$, respectively. They also suggested HA as predictor of severe fibrosis capable of reflecting the degree and severity of hepatic fibrosis. In this study, the mean serum level of HA in patients with mild fibrosis was reported as $22 \mathrm{ng} / \mathrm{mL}$, while it was 118 $\mathrm{ng} / \mathrm{mL}$ in patients with severe fibrosis, and there was statistically significance. Orasan et al. ${ }^{(3)}$ had the same opinion. They observed that HA levels were 
significantly higher in patients with NASH and fibrosis from those with NASH and without fibrosis, while also correlating with each stage of fibrosis. The best cut-off value was at $148.8 \mathrm{ng} / \mathrm{mL}$ for the identification of patients with NAFLD and fibrosis (sensitivity was $95.7 \%$, specificity $96.3 \%$, PPV $95.7 \%$, NPV $96.3 \%$ and accuracy of $96 \%$ higher than that of LN). Similarly, in the study by Dvorak et al. (19), HA was the most significant single biochemical factor to discriminate mild and moderate fibrosis against significant fibrosis ( $\mathrm{AUC}=0.94$ ).

On the other hand, the study of Angulo disagreed with this result. He found that HA is less accurate for detecting lesser degrees of fibrosis with an AUROC for any degree of fibrosis varying between 0.67 and 0.73 . In addition, HA increases in any systemic inflammatory condition, which may produce false positive predictive results ${ }^{(20)}$.

\section{CONCLUSION}

To our knowledge, and according to this study's results, HA can be reliably used as an accurate and specific non-invasive biomarker for the diagnosis of NAFLD and staging of the severity of disease compared with the traditional known non-invasive scores.

\section{REFERENCES}

1. Byrne C, Targher G (2015): NAFLD: A multisystem disease. J Hepatol., 62: 47-64.

2. Kaneda H, Hashimoto E, Yatsuji S et al. (2016): Hyaluronic acid levels can predict severe fibrosis and platelet counts can predict cirrhosis in patients with nonalcoholic fatty liver disease. J Gastroenterol Hepatol., 21(9):1459-65.

3. Orasan O, Ciulei G, Cozma A et al. (2016): Hyaluronic acid as a biomarker of fibrosis in chronic liver diseases of different etiologies. Clujul Med., 89(1):24-31.

4. Loaeza-del-Castillo A, Paz-Pineda F, OviedoCárdenas E et al. (2008): AST to platelet ratio index (APRI) for the non-invasive evaluation of liver fibrosis. Ann Hepatol., 7(4):350-7.

5. Papagianni M, Sofogianni A, Tziomalos K (2015): Non-invasive methods for the diagnosis of nonalcoholic fatty liver disease. World J Hepatol., 7(4):638-48.

6. Bedogni G, Kahn H S, Bellentani S et al. (2014): A simple index of lipid overaccumulation is a good marker of liver steatosis', BMC Gastroenterology. BioMed Central, 10(1):1-8.

7. McPherson S, Hardy T, Dufour J et al. (2017): Age as a confounding factor for the accurate non-invasive diagnosis of advanced NAFLD fibrosis. Am J Gastroenterol., 112(5):740-751.

8. Gudowska M, Gruszewska E, Panasiuk A et al. (2016): Hyaluronic acid concentration in liver diseases. Clin Exp Med., 16(4):523-528.

9. Suzuki A, Angulo P, Lymp J et al. (2005): Hyaluronic acid, an accurate serum marker for severe hepatic fibrosis in patients with non-alcoholic fatty liver disease. Liver International, 25(4): 779-786.

10. Hadizadeh F, Faghihimani E, Adibi P (2017): Nonalcoholic fatty liver disease: Diagnostic biomarkers. World J Gastrointest Pathophysiol., 8(2): 11-26.

11. Martin-Rodriguez J, Cantero J, Cantero A et al. (2017): Diagnostic accuracy of serum alanine aminotransferase as biomarker for nonalcoholic fatty liver disease and insulin resistance in healthy subjects, using 3T MR spectroscopy. Medicine (Baltimore), 96(17):6770-5.

12. Khosravi S, Alavian S, Zare A et al. (2011): Nonalcoholic fatty liver disease and correlation of serum alanin aminotransferase level with histopathologic findings. Hepat Mon., 11(6):452-8.

13. Zubair R, Mirza M, Iftikhar J et al. (2018): Frequency of incidental fatty liver on ultrasound and its association with diabetes mellitus and hypertension. Pak J Med Sci. , 34(5):1137-1141.

14. Zhang Z, Wang J, Wang $H$ (2018): Correlation of blood glucose, serum chemerin and insulin resistance with NAFLD in patients with type 2 diabetes mellitus. Exp Ther Med., 15(3):2936-2940.

15. Mahaling D, Basavaraj M, Bika A (2013): Comparison of lipid profile in different grades of non-alcoholic fatty liver disease diagnosed on ultrasound. Asian Pac J Trop Biomed. , 3(11): 907-912.

16. Dyson J, Anstee Q, McPherson S (2014): Nonalcoholic fatty liver disease: a practical approach to diagnosis and staging. Frontline Gastroenterol., 5(3):211-218.

17. Yousaf M, Chaudhary F, Mehal W (2019): The impact of scoring system in the evaluation of Nonalcoholic fatty liver disease ( NAFLD ) and nonalcoholic steatohepatitis ( NASH ). Gastroenterol Hepatol Open Access, 10(5):258-260.

18. Kupčová $V$, Fedelešová $M$, Bulas $J$ et al. (2019): Overview of the pathogenesis, genetic, and non-invasive clinical, biochemical, and scoring methods in the assessment of NAFLD. Int J Environ Res Public Health, 16(19):3570-3.

19. Dvorak K, Stritesky J, Petrtyl J et al. (2014): Use of non-invasive parameters of non-alcoholic steatohepatitis and liver fibrosis in daily practice - An exploratory casecontrol study. PLoS One, 9(10): 111551-6.

20. Angulo $P$ (2009): Noninvasive assessment of fibrosis and steatosis in NASH and ASH. Gastroentérologie Clinique et Biologique, 33:940-8. 